\title{
Binocular open-view system to perform estimations of aberrations and scattering in the human eye
}

\author{
Carlos E. García-Guerra ${ }^{1,}$, Mikel Aldaba $^{1}$, Montserrat Arjona ${ }^{1}$, and Jaume Pujol ${ }^{1}$ \\ ${ }^{1}$ Centre of Sensors, Instruments and Systems Development (CD6). Universitat Politècnica de Catalunya (UPC). Rambla Sant Nebridi 10, 08222, Terrassa \\ (Barcelona), Spain. \\ *Corresponding author: carlos.enrique.garcia@cd6.upc.edu \\ Compiled January 20, 2016
}

\begin{abstract}
We present a system that integrates a double-pass (DP) instrument and a Hartmann-Shack (HS) wavefront sensor to provide information not only on aberrations, but also on scattering occurring in the human eye. A binocular open-view design permits evaluations in normal viewing conditions. Furthermore, the system is able to compensate for both spherical and astigmatic refractive errors during measurements by using devices with configurable optical power. The DP and HS techniques provide comparable data after estimating wavefront slopes with respect to intersections of an ideal grid and compensating for residual errors provoked by optical defects of the measuring system. Once comparable data is obtained, it is possible to use this combined manner of assessment to provide information on scattering. Measurements in an artificial eye suggest that the characteristics of the ocular fundus may induce deviations of DP with respect to HS data. These differences were quantified in terms of the modulation transfer function in young healthy eyes measured in infrared light to demonstrate the potential use of the system in visual optics studies.
\end{abstract}

OCIS codes: (330.7325) Visual optics, metrology; (330.1400) Vision - binocular and stereopsis.

http://dx.doi.org/10.1364/ao.XX.XXXXXX

\section{INTRODUCTION}

The optical characterization of the eye is of great interest because it offers a tool to describe and understand different phenomena arising from the interaction of light with the human eye. In this context, double-pass (DP) instruments [1] and Hartmann-Shack (HS) sensors [2] are complementary technologies used in clinical practice $[3,4]$ to describe optical properties of the eye. While in DP instruments the aerial retinal image is used to compute optical quality descriptors, in HS sensors the wave aberration is reconstructed from estimations of wavefront slopes at a plane conjugate to the pupil of the eye.

Under conventional data analysis, both the DP and the HS technique provide information on aberrations [5]. Additionally, double-pass data contain the effects of scattering [5-7]. In the case of the ocular fundus, this phenomenon may arise from the interaction of light with the retina and deeper layers up to which certain wavelengths may penetrate [8-10]. In addition, the quantification of scattering from DP images may be affected by the presence of aberrations [11]. In this sense, instruments combining multiple assessment modes and providing comparable data would improve the quantification of phenomena such as the scattering, thus overcoming the limitations of conventional DP and HS data processing when the instruments work independent of each other.

Different authors have presented instruments that implement multiple techniques. For instance, Prieto et al. and Aldaba et al. reported systems combining DP and HS instruments to validate aberrometry estimations [12] and to study accommodation [13]. However, in neither of these two works there is a combined data analysis to provide complementary information on the optical quality. On the other hand, Rodriguez and Navarro employed a single system to quantify intraocular scattering from differences between DP estimations and those obtained with a laser ray tracing system [14]. These measurements were performed using green light, which may result uncomfortable for the patients due to brightness. The scattering process occurring in the ocular fundus has been also considered in visual optics, but only to explain differences between DP data and that obtained with instruments that are not affected by scattering, such as interferometric devices [9] and HS sensors [7]. Although these examples of systems based on multiple techniques, it is noticeable that they 
correspond to monocular systems. When binocular instruments have been implemented, they have relied on single technologies [15-19].

With the idea of performing a comprehensive evaluation of the optical quality, we have designed, implemented, and validated a system that combines a DP instrument and a HS sensor. In this manner, the system is capable of providing not only overall estimations on the optical quality and description on aberrations, but also information on scattering through a combined data analysis. A binocular open-view design permits measurements during normal viewing conditions. Additionally, the instrument uses devices of tunable optical powers to compensate for spherical and astigmatic refractive errors. Since the implemented system works in infrared light, differences between techniques may provide information on layers of the ocular fundus beyond the retina, such as the choroid, up to which light may penetrate in that part of the spectrum [9]. In this manner, the system could be used to study not only the impact of the ocular fundus in double-pass measurements at the working wavelength, but also to characterize the behavior of the scattering from the ocular fundus in a healthy population. This information could be then used to detect deviations with respect to average values as an indicator of possible problems in the choroid.

This article introduces first the system and the methodology that is followed to obtain comparable DP and HS data. It then presents measurements on an artificial eye using diffusers with different characteristics to illustrate the impact of the ocular fundus on DP and HS measurements. Finally, preliminary data from measurements on real eyes are presented to show the potential use of the system in quantifying scattering.

\section{THE BINOCULAR OPEN-VIEW SYSTEM}

\section{A. Apparatus}

The system is shown in figure 1. Collimated light from a super luminescent diode SLD (Superlum SLD-37-HP2-DIL-SM-PD, $\lambda=801 \mathrm{~nm}$ ) is divided into two beams by mask $P_{1}$, which is composed of two horizontally-aligned apertures of $2 \mathrm{~mm}$ in diameter and $8 \mathrm{~mm}$ in center-to-center separation. After being reflected by beam splitters $B S_{1}$ and $B S_{2}$ and passing through the telescopic system formed by lenses $L_{1}$ and $L_{2}$ of $f^{\prime}=200 \mathrm{~mm}$ in focal lengths, the beams are separated by reflections on mirror $M_{1}$. Then, light going to the left and right eye is processed by two independent branches of identical optical elements. The light is compensated first for astigmatism using $C_{1-2}$ by controlling the angle between two identical ophthalmic lenses of $S=+1 \mathrm{D}$ and $C=-2 \mathrm{D}$ in spherical and astigmatic prescription [20]. The commercially available remote-controlled focus-tunable lens $L_{T}$ (Optotune EL-10-30-NIR-LD, $f^{\prime} \approx 45-120 \mathrm{~mm}$ ) is used in combination with lens $L_{C}$ of fixed focal length $\left(f^{\prime}=75 \mathrm{~mm}\right)$ to compensate for spherical refractive errors [21]. The distance between lenses $L_{T}$ and $L_{C}$ and between $L_{C}$ and the pupil plane is twice the focal distance of lens $L_{C}$, so that $L_{T}$ is also in a plane conjugated to the pupil of the eye. Mirrors $M_{2}, D M$, and $H M$ are used to direct light to the eye. In addition, the dichroic mirror $D M$ permits camera $C M_{P}$ to monitor the position of the pupil of the eye for alignment purposes. On the other hand, transverse displacements and angular rotations of hot mirrors $H M$ are used to control the interpupillary distance and the angle of incidence of light, respectively.

After entering the eye, light is focused on the ocular fundus. Following an optical path identical to that of the first pass, light

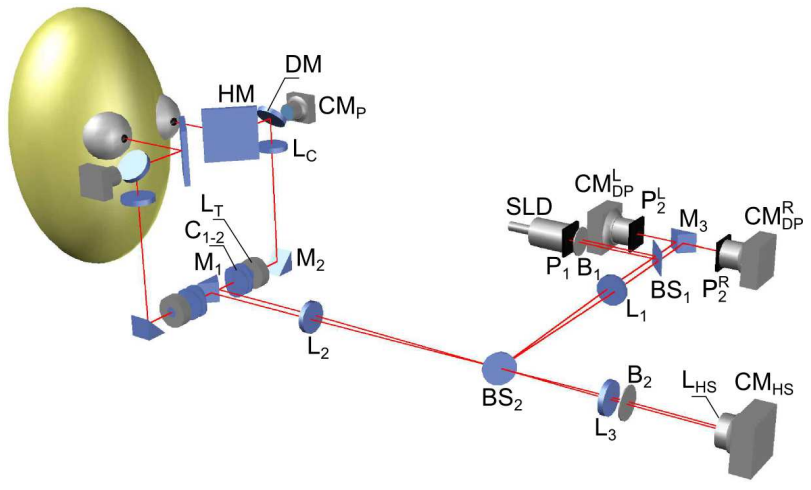

Fig. 1. Scaled representation of the implemented system. Light source: $S L D$; apertures: $P_{1}, P_{2}$; polarizers: $B_{1}, B_{2}$; beam splitters: $B S_{1}, B S_{2}$; lenses: $L_{1}, L_{2}, L_{3}$; mirrors: $M_{1}, M_{2}, M_{3}$; tunable devices: $C_{1-2}, L_{T}-L_{C}$; dichroic mirror: $D M$; hot mirror: $H M$; lenslet array: $L_{H S}$; cameras: $C M_{P}, C M_{D P}, C M_{H S}$.

resulting from the reflection process in the fundus of both eyes is redirected by $M_{1}$ towards the telescopic systems $L_{2}-L_{1}$ and $L_{2}-L_{3}$. In the former case, light reflected by $B S_{2}$, transmitted by $B S_{1}$, and redirected by prism mirror $M_{3}$ reaches circular apertures $P_{2}^{L}$ and $P_{2}^{R}$ of $4 \mathrm{~mm}$ in diameter. These apertures act as exit pupils and are located in front of cameras $C M_{D P}^{L}$ and $C M_{D P}^{R}$ (IDS UI-1240ML-NIR), which record, respectively, DP retinal images of the left and right eye both using objectives of $f^{\prime}=100 \mathrm{~mm}$. In the case of the HS configuration, light transmitted by $B S_{2}$ is refracted by lens $L_{3}$ of $100 \mathrm{~mm}$ in focal length and filtered by linear polarizer $B_{2}$ that in combination with the crossed polarizer $B_{1}$ located in front of the laser source allows elimination of corneal reflection in HS images [22]. This configuration permits the use of a single micro-lens HS array $L_{H S}$ (AOA 0200-6.3-S-C) followed by camera $C M_{H S}$ (IDS UI-1241LE-NIR-GL) to sample the pupils of both eyes simultaneously.

\section{B. Calibration process}

There is a series of factors that may induce aberrations in the implemented system. For instance, some optical devices have been tilted to avoid reflections within the second-pass path of light, which induces astigmatism. While they are always present in DP measurements, the effects of aberrations may be masked in HS estimations if they are contained in the data used as reference during the computation of wave aberrations. Conventionally, wavefront slopes are computed with respect to centroids positions in a reference image, which includes deviations provoked by the instrument [2]. In order to have HS and DP data with the same information on aberrations, including the ones of the measurement instrument, the implemented system measures spot displacements from theoretical positions, a pattern of equally spaced horizontal and vertical lines whose intersections indicate the positions where the spots would incise in an aberration-free system.

Differences arising from the non-common path between techniques, higher order aberrations not included during wavefront reconstruction and possible diffusion in the optical fluid forming the focus-tunable lens may provoke deviations between DP and HS data. Considering a multiplicative model, the modulation transfer function (MTF) for the image with the best possible optical quality could be expressed as $M T F_{B}=M T F_{D L} \times M T F_{R}$, where the latter two terms represent the diffraction limited 
response and the contribution of residual errors provoked by the system, respectively. Since the theoretical performance of $M T F_{D L}$ is known for a given pupil diameter [23], it is possible to quantify the contribution of residual errors by filtering the effects of diffraction from $M T F_{B}$. In turn, this information may be used to provide the response of the eye as $\widehat{M T F}$ by using equation 1, which represents an estimation of any measured $M T F_{M}$ after being compensated for residual errors.

$$
\widehat{M T F}=\frac{M T F_{M}}{M T F_{R}}
$$

This method of compensation was applied in the implemented system over both DP and HS data. Curves $M T F_{B}$ were obtained for both techniques during the calibration process from images for the best optical quality (narrowest spot in the DP sensor) when the system was irradiated with collimated light from the eye's pupil plane. The first pass response can be considered diffraction limited due to its small pupil size. For this reason, $M T F_{R}$ was estimated only for the second pass pupil size. Once determined, the corresponding contribution of residual errors was used in all subsequent measurements to compensate for its effects during DP and HS data processing by filtering $M T F_{R}$ in any measured data. Thus, the MTF curves presented hereafter correspond in reality to $\widehat{M T F}$, the estimated versions of those responses computed directly from the recorded images.

\section{System validation}

The proposed method of compensation was verified by comparing DP with HS responses. Although similar results were obtained for both the right-eye and left-eye optical paths of the system, we present here only results for the latter. The curves were obtained from the average of 5 consecutive images for the best optical quality. It was done for an induced astigmatism and defocus of $-0.25 D$ both while the system was irradiated with collimated light. The induced aberrations were generated by placing trial lenses in front of the light source.
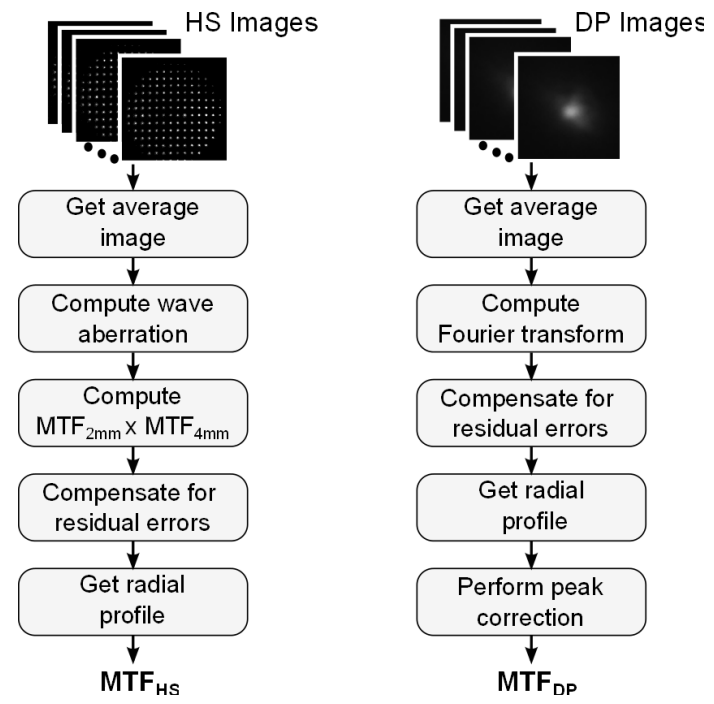

Fig. 2. Flow diagram of the data processing that was followed to compute the HS (left) and DP (right) MTF.

The MTF curves were computed using Matlab (Mathworks, 2010) in the following manner. In the case of double-pass data, the average image was Fourier transformed and corrected for residual errors of the measuring system. Then, the MTF was obtained after proper normalization by taking the radial profile of the compensated spectral representation and applying a peak correction [24] by extrapolating lower frequencies from a curve fitting based on exponential functions [25]. When working with HS data, the wave aberration was estimated using 54 Zernike coefficients. Then, the MTF was computed as the autocorrelation of the complex pupil functions [23] for the first and second pass pupil diameters $\left(M T F_{2 \mathrm{~mm}} \times M T F_{4 \mathrm{~mm}}\right)$. As in the case of DP processing, HS data was compensated for residual errors before computing the radial profile of the MTF. This process is illustrated in figure 2 and represents the methodology to compute the MTF curves presented in this work.

The resultant curves are plotted in figure 3. As observed, comparable DP and HS curves are obtained when wavefront slopes are computed with respect to an ideal grid acting as a reference and when there is a compensation for residual errors.

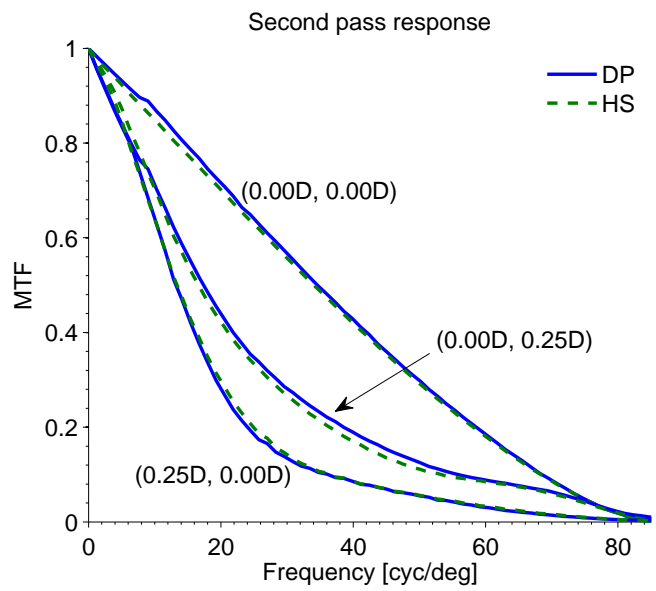

Fig. 3. Second pass response of the system for the DP (solid line) and HS (dashed line) sensors for different induced aberrations labeled as (sphere, astigmatism).

\section{EFFECTS OF THE OCULAR FUNDUS IN DP AND HS MEASUREMENTS}

Once corroborated that the system provides comparable DP and HS data, the MTF was measured for an artificial eye. Such a device consisted of a lens of $50 \mathrm{~mm}$ in focal length and its response was obtained for two cardboards $\left(R_{1}\right.$ and $\left.R_{2}\right)$ of different roughness acting as the ocular fundus. Quantified with the 3D profiler PLu apex (Sensofar) [26], the cardboards presented height fluctuations (standard deviations) of 3.984 and $0.900 \mu \mathrm{m}$ in diffusers $R_{1}$ and $R_{2}$, respectively. Thus, roughness in $R_{1}$ is higher than in $R_{2}$. The interchangeable diffuse materials were mounted in a rotating motor to break light coherence [27] by producing uncorrelated patterns during image recording. In this manner, the saved frames were not affected by speckle. Using an integration time of $40 \mathrm{~ms}, 5$ images were recorded for both the double-pass and the Hartmann-Shack sensor. Then, the average image was used to compute the MTF using both techniques.

Figure 4 depicts the DP and HS MTF for both diffuse materials. As observed, the HS curves are practically overlapped for both of the diffusers acting as the ocular fundus. On the contrary, double-pass data present a variability, which may be provoked by differences in the properties between $R_{1}$ and $R_{2}$. The higher magnitude of the deviations for retina $R_{1}$ with respect to those 
for $R_{2}$ may be related to the larger height fluctuations of that diffuse material. These results are in agreement with those presented in [2] and [9], which indicate that DP could be affected by the scattering arising from the interaction of light with the ocular fundus.

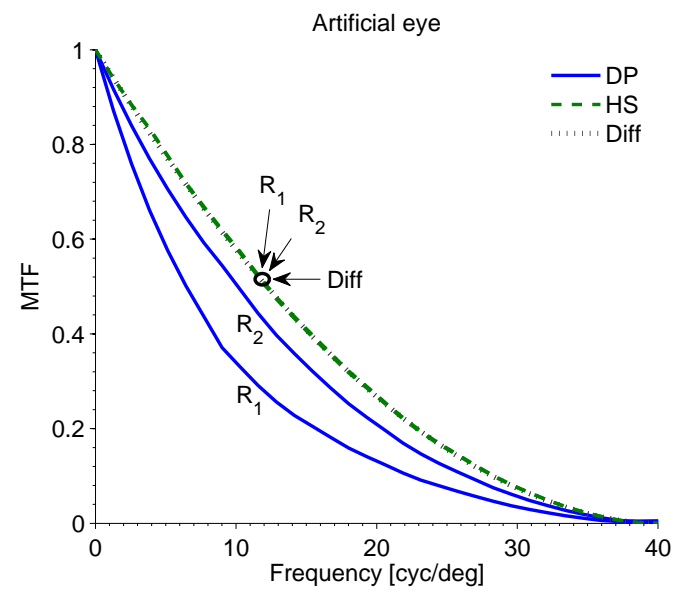

Fig. 4. DP (solid line) and HS (dashed line) response for an artificial eye using retinas $R 1$ and $R 2$. The double-pass diffraction limited curve is plotted in the figure (dotted line), although it is practically overlapped by HS data.

The results presented in this section indicates on the one hand that double-pass systems detect scattering arising in the ocular fundus and on the other hand that multimodal systems based on DP and HS measurements can be used to quantify this phenomenon. This quantification can be based, for instance, on differences between curves, as done by other authors [5, 28].

\section{DP AND HS PERFORMANCE IN REAL EYES}

In order to quantify the performance in real eyes, 8 young healthy left eyes were measured while patients were binocularly looking at an external fixation target located at a distance of six meters. The mean age of the subjects was $26 \pm 4$ years old. 5 series of 20 images were recorded for each subject after setting up properly both the spherical and the astigmatic refractive error corrector and the power of the laser source. All the images were recorded using an integration time of $40 \mathrm{~ms}$. The series of recordings were performed for each subject in a consecutive manner in a single session and under similar environment illumination conditions. In general, the five series of measurements were carried out in less than 10 minutes.

During data processing, the 20 DP and HS images belonging to a series were averaged. In this manner, the speckle noise affecting the individual short-exposure recordings was eliminated. The resulting images were used to estimate a DP and a HS MTF per series by following the procedure depicted in figure 2 . At the end of this process, $5 \mathrm{DP}$ and 5 HS estimations were available per measured eye. After this, the average curve of the $5 \mathrm{MTF}$ estimations was used to compute the difference between the DP and the HS MTF.

Figure 5 shows the average DP and HS MTF of all subjects. The lower magnitude is again observed for DP with respect to HS estimations. For instance, the magnitude at $10 \mathrm{cyc} / \mathrm{deg}$ of the HS MTF is around twice that for the DP average curve. This behavior was expected for several reasons. Among other factors, the system works in infrared light, which is known to be affected by scattering arising from layers of the fundus beyond the retina [29]. Moreover, HS measurements do not provide information on this phenomenon [5]. On the other hand, wave aberrations were computed using nine order (54) Zernike coefficients. Since the majority of the higher order aberrations are described with eight order (42) Zernike coefficients [7], we assume that the deviations are not provoked by higher order aberrations in HS data. Taking into account that measurements were performed in young healthy subjects, the effects of intraocular scattering can be considered negligible.

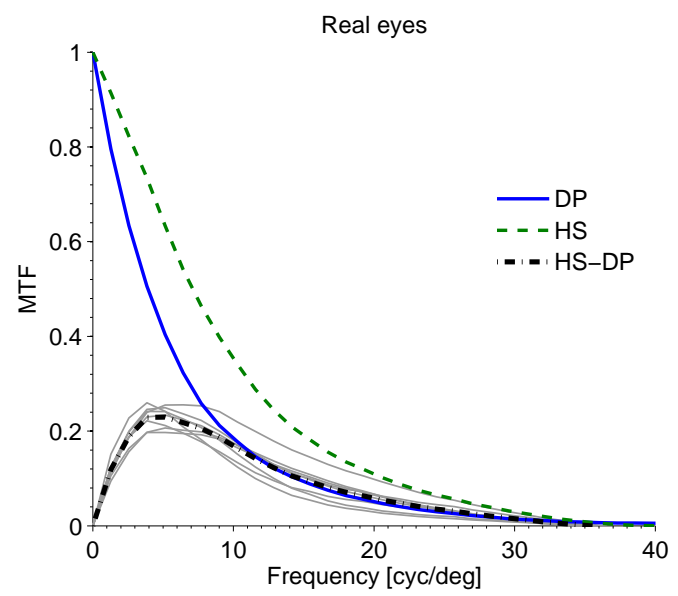

Fig. 5. Average DP (solid line) and HS (dashed line) response for 8 real eyes. The average difference between HS and DP data is plotted in the figure (black dashed-dotted line), as well as their individual estimations (gray solid line).

The differences between DP and HS data are also plotted in figure 5. They present similar behaviors for all measured subjects: a curve with a peak of around 0.20 in magnitude at 5 cyc/deg. Let us consider the hypothetical situation in which all eyes had been affected by the same scattering, but by different aberrations. Under this condition, the differences between MTF curves would remain identical for all cases, regardless of the effects on $M T F_{D P}$ and $M T F_{H S}$ attributed to differences in aberrations. Assuming that the measuring system provides comparable data in cases without scattering, the variations among the individual estimations observed in the figure may be attributed to changes in the amount of scattering light among the measured eyes.

If DP and HS differences contain information on the characteristics of the retina, as corroborated for the artificial eye, the multimodal system presented in this work might help to perform precise comparison between techniques in studies on scattering. Moreover, deviations from average values could be used to detect conditions affecting the retina. However, further research is needed in this direction.

\section{CONCLUSIONS}

This article presented a binocular open-view system that implements a DP and a HS configuration to measure the eye's optical quality in normal viewing conditions. The system is able to compensate for both astigmatic and spherical refractive errors. During data processing, wavefront slopes were estimated as deviations from intersections of an ideal grid, while residual errors were compensated for with respect to reference images. Under these conditions, comparable DP and HS data were obtained. 
Measurements in an artificial eye using retinas with different characteristics corroborates that DP data contains information on retinal scattering. These differences were quantified in real eyes, whose behaviors were similar in all cases. Thus, a characterization of the differences could be used to detect conditions affecting the ocular fundus. The results presented here show the potential use of the system to perform studies in visual optics.

\section{ACKNOWLEDGMENT}

The authors would like to thank Cristina Martínez for her help in the measurement of the paper roughness and the Ministerio de Economía y Competitividad for his support to this project through the grant DPI2014-56850-R. Carlos E. García-Guerra acknowledges the fellowship within the framework of the joint doctorate program Europhotonics between Polytechnic University of Catalonia (UPC, Spain) and Karlsruhe Institute of Technology (KIT, Germany).

\section{REFERENCES}

1. J. Santamaría, P. Artal, and L. Bescós, "Determination of the pointspread function of human eyes using a hybrid optical-digital method," J. Opt. Soc. Am. A 4, 1109-1114 (1987).

2. J. Liang, B. Grimm, S. Goelz, and J. F. Bille, "Objective measurement of wave aberrations of the human eye with the use of a Hartmann-Shack wave-front sensor," J Opt. Soc. Am. A 11, 1949-1957 (1994).

3. J. L. Güell, J. Pujol, M. Arjona, F. Díaz-Doutón, and P. Artal, "Optical Quality Analysis System; Instrument for objective clinical evaluation of ocular optical quality," J. Cataract. Refract. Surg. 4, 1109-1114 (1987).

4. J. J. Rozema, D. E. M. V. Dyck, and M.-J. Tassignon, "Clinical comparison of 6 aberrometers. part 1: Technical specifications," J. Cataract Refract. Surg. 31, 1114-1127 (2005).

5. F. Díaz-Doutón, A. Benito, J. Pujol, M. Arjona, J. L. Güell, and P. Artal, "Comparison of the Retinal Image Quality with a Hartmann-Shack Wavefront Sensor and a Double-Pass Instrument," IOVS 47, 17101726 (2006).

6. G. Westheimer and J. Liang, "Influence of ocular light scatter on the eye's optical performance," J. Opt. Soc. Am. A 12, 1417-1424 (1995).

7. J. Liang and D. R. Williams, "Aberrations and retinal image quality of the normal human eye," J. Opt. Soc. Am. A 14, 2873-2883 (1997).

8. P. Artal and R. Navarro, "Simultaneous measurement of two-pointspread functions at different locations across the human fovea," Applied Optics 39, 3646-3656 (1992).

9. D. R. William, D. H. Brainard, M. J. McMahon, and R. Navarro, "Doublepass and interferometric measures of the optical quality of the eye," $\mathrm{J}$. Opt. Soc. Am. A 11, 3123-3435 (1994).

10. I. J. Hodgkinson, A. C. B. Molteno, and P. B. Greer, "Point-spread function for light scattered in the human ocular fundus," J. Opt. Soc. Am. A 11, 479-486 (1994).

11. P. Artal, A. Benito, G. M. Pérez, E. Alcón, A. de Casas, J. Pujol, and J. M. Marín, "An objective scatter index based on double-pass retinal images of a point source to classify cataracts," PLoS ONE 6, e16823 (2011).

12. P. M. Prieto, F. Vargas-Martín, S. Goelz, and P. Artal, "Analysis of the performance of the Hartmann-Shack sensor in the human eye," J. Opt. Soc. Am. A 17, 1388-1398 (2000).

13. M. Aldaba, M. Vilaseca, F. Díaz-Doutón, M. Arjona, and J. Pujol, "Measuring the accommodative response with a double-pass system: Comparison with the hartmann-shack technique," Vision 62, 26 - 34 (2012).

14. P. Rodríguez and R. Navarro, "Double-pass versus aberrometric modulation transfer function in green light," Journal of Biomedical Optics 12, 044018 (2007).

15. E. Chirre, P. M. Prieto, and P. Artal, "Binocular open-view instrument to measure aberrations and pupillary dynamics," Optics Letters 39, 4773-4775 (2014).
16. R. Sabesan, L. Zheleznyak, and G. Yoon, "Binocular visual performance and summation after correcting higher order aberrations," Biomedical Optics Express 3, 3176-3789 (2012).

17. E. J. Fernández, P. M. Prieto, and P. Artal, "Binocular adaptive optics simulator," Optics Letters 34, 2628-2630 (2009).

18. S. S. Chin, K. M. Hampson, and E. A. H. Mallen, "Binocular correlation of ocular aberrations dynamics," Optics Express 16, 14731-14745 (2008).

19. M. Kobayashi, N. Nakazawa, T. Yamaguchi, T. Otaki, Y. Hirohara, and T. Mihashi, "Binocular open-view shack-hartmann wavefront sensor with consecutive measurements of near triad and spherical aberration," Applied Optics 47, 4619 (2008).

20. J. Arines and E. Acosta, "Low-cost adaptive astigmatism compensator for improvement of eye fundus camera," Optics Letters 36, 4164-4166 (2011).

21. F. Sanàbria, F. Díaz-Doutón, M. Aldaba, and J.Pujol., "Spherical refractive correction with an electro-optical liquid lens in a double-pass system," J. Europ. Opt. Soc. Rap. Public. 8, 13062 (2013).

22. S. Marcos, L. Diaz-Santana, L. Llorente, and C. Dainty, "Ocular aberrations with ray tracing and Shack-Hartmann wave-front sensors: Does polarization play a role?" J. Opt. Soc. Am. A 19, 1063-1072 (2002).

23. J. W. Goodman, Introduction to Fourier Optics (McGraw-Hill, 1998), 2nd ed.

24. A. Guirao, C. González, M. Redondo, E. Geraghty, S. Norrby, and P. Artal, "Average Optical Performance of the Human Eye as a Function of Age in a Normal Population," IOVS 40, 203-213 (1999).

25. P. Artal and R. Navarro, "Monochromatic modulation transfer function of the human eye for different pupil diameters: an analytical expression," J. Opt. Soc. Am. A 11, 246-249 (1994).

26. A. Pintó, F. Laguarta, R. Artigas, and C. Cadavall, "Non-contact measurement of aspherical and freeform optics with a new confocal tracking profiler," in "Optical Fabrication, Testing, and Metrology IV," , vol. 8169 (2011), vol. 8169, p. 81690V.

27. E. Moreno-Barriuso and R. Navarro, "Laser Ray Tracing versus Hartmann-Shack sensor for measuring optical aberrations in the human eye," J. Opt. Soc. Am. A 17, 974-985 (2000).

28. M. Shahidi and Y. Yang, "Measurements of ocular aberrations and light scatter in healthy subjects," Optometry and Vision Science 81, 853-857 (2004).

29. H. S. Ginis, G. M. Pérez, J. M. Bueno, A. Pennos, and P. Artal, "Wavelength dependence of the ocular straylight," IOVS 54, 3702-3708 (2013). 